\title{
Coactivator cross-talk specifies transcriptional output
}

\author{
Michael T. Marr II, Yoh Isogai, Kevin J. Wright, and Robert Tjian ${ }^{1}$ \\ Howard Hughes Medical Institute, Department of Molecular and Cell Biology, University of California, \\ Berkeley, California 94720, USA
}

\begin{abstract}
Cells often fine-tune gene expression at the level of transcription to generate the appropriate response to a given environmental or developmental stimulus. Both positive and negative influences on gene expression must be balanced to produce the correct level of mRNA synthesis. To this end, the cell uses several classes of regulatory coactivator complexes including two central players, TFIID and Mediator (MED), in potentiating activated transcription. Both of these complexes integrate activator signals and convey them to the basal apparatus. Interestingly, many promoters require both regulatory complexes, although at first glance they may seem to be redundant. Here we have used RNA interference (RNAi) in Drosophila cells to selectively deplete subunits of the MED and TFIID complexes to dissect the contribution of each of these complexes in modulating activated transcription. We exploited the robust response of the metallothionein genes to heavy metal as a model for transcriptional activation by analyzing direct factor recruitment in both heterogeneous cell populations and at the single-cell level. Intriguingly, we find that MED and TFIID interact functionally to modulate transcriptional response to metal. The metal response element-binding transcription factor-1 (MTF-1) recruits TFIID, which then binds promoter DNA, setting up a "checkpoint complex" for the initiation of transcription that is subsequently activated upon recruitment of the MED complex. The appropriate expression level of the endogenous metallothionein genes is achieved only when the activities of these two coactivators are balanced. Surprisingly, we find that the same activator (MTF-1) requires different coactivator subunits depending on the context of the core promoter. Finally, we find that the stability of multi-subunit coactivator complexes can be compromised by loss of a single subunit, underscoring the potential for combinatorial control of transcription activation.
\end{abstract}

[Keywords: Coactivator; MTF-1; RNAi; TFIID; mediator]

Received February 9, 2006; revised version accepted March 20, 2006.

In order for cells to survive, they must be able to interpret environmental and developmental signals. Often the critical targets of these signals are sequence-specific DNA-binding proteins that serve as transcriptional activators or repressors. These relatively small regulatory proteins bind DNA at their cognate promoters to modulate the specific patterns of gene expression needed to respond to particular signals. However, these transcription factors that bind promoter DNA are generally not able to potentiate transcriptional readouts on their own (Hoffman et al. 1990; Peterson et al. 1990; Ryu et al. 1999). Instead, the tight control of gene expression is often dependent on the ordered assembly of large multiprotein coregulators at the promoters of mRNA genes that respond to specific cellular signals (Dynlacht et al. 1991; Naar et al. 1998; Fondell et al. 1999; Rachez et al. 1999; Ryu et al. 1999).

These multiprotein complexes can be divided, albeit roughly, into several distinct classes based on their prop-

${ }^{1}$ Corresponding author.

E-MAIL jmlim@uclink4.berkeley.edu; FAX (510) 643-9547.

Article is online at http://www.genesdev.org/cgi/doi/10.1101/gad.1418806. erties (Lemon and Tjian 2000; Naar et al. 2001; Taatjes et al. 2004). One class, the coactivators, interprets activator signals and conveys them to the basal transcription apparatus. Of these, TFIID and Mediator (MED) are known to be direct targets of sequence-specific DNA-binding proteins. TFIID has been shown to be required for activated transcription at many promoters (Naar et al. 2001). TFIID consists of the TATA-binding protein (TBP) and the TBP-associated factors (TAFs). The central components of the complex are conserved from yeast to man. However, the metazoan version of TFIID has expanded to include some important differences. For instance, the glutamine-rich amino terminus of TAF4, a common target of activators, is missing in the yeast homolog. Additionally, metazoans have developed tissue-specific versions of the TAFs that play a role in gonad function, further expanding the repertoire of coactivators (Dikstein et al. 1996; Freiman et al. 2001; Hiller et al. 2004). Likewise, many subunits of the MED complex are conserved from yeast to man. However, the MED complex has diverged over time. The regions of obvious similarity in pairwise alignments of the subunits are often small, 
with large insertions and expansions occurring in the metazoan homologs (Boube et al. 2002). Moreover, there are at least 11 metazoan-specific subunits, and recent analysis of the mammalian MED complex revealed that it too contains potentially gene-specific variant subunits, although the role of these subunits remains unclear (Sato et al. 2004).

Many coactivator complexes have been identified based on their ability to stimulate activated transcription in defined in vitro systems (Meisterernst et al. 1991; Kim et al. 1994; Naar et al. 1998; Rachez et al. 1999; Ryu et al. 1999). Interestingly, although both TFIID and MED are targets for activators, many promoters require both coactivators in vitro. This suggests that the complexes are not redundant but may instead perform nonoverlapping functions in potentiating transcription. Furthermore, in vitro experiments with a synthetic activator suggest that TFIID and MED might functionally interact (Johnson et al. 2002; Johnson and Carey 2003). Exactly what role each of the complexes plays remains an open question. Moreover, it is unclear whether these complexes also interact with each other to cross-talk during the process of transcriptional regulation in vivo and whether their function has diversified in different metazoan cells.

Here, we examine the potential functional interactions and specific role of the TFIID and MED complexes at the Drosophila metallothionein promoters in vivo by taking advantage of the efficient RNAi response in insect cells (Clemens et al. 2000). We have depleted various subunits of the MED and TFIID complex by RNAi and examined the effect of the loss of these subunits on both the level of mRNA synthesized and the physical recruitment of the transcriptional apparatus in conventional cell population experiments as well as in single cells. We find that the two coactivator complexes interact to meter the transcriptional response so that it is physiologically appropriate. Unexpectedly, we found that although depleting MED subunits compromises the metal inducible activation of transcription, depleting both TFIID and MED components restores much of the transcriptional activity of the metallothionein A gene, suggesting an intriguing functional relationship between the TAFs and MED coactivator complexes.

\section{Results}

The metallothionein A (MtnA) gene as a model for gene activation

There are four known metallothionein genes in Drosophila: MtnA, MtnB, MtnC, and MtnD (Egli et al. 2003). Of these, the best characterized is the MtnA gene (Fig. 1A), which produces a transcript of $\sim 600$ bases in length, bearing one intron. All of the regulatory elements required for robust response to heavy metals, including copper, lie within 500 bp of the transcription start site (Maroni et al. 1986, 1987; Otto et al. 1987). The gene is controlled by a single activator, metal response elementbinding transcription factor 1 (MTF-1), which binds two
A

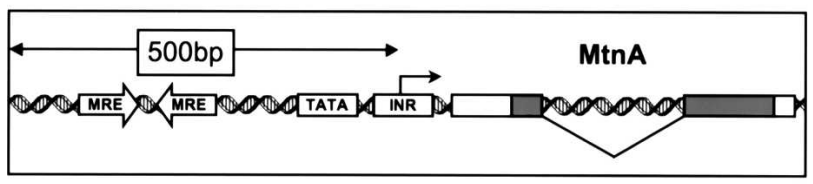

B

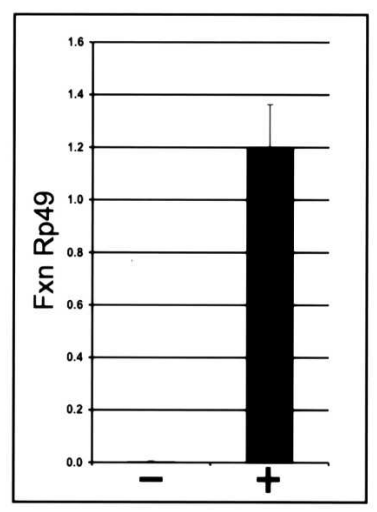

C

D

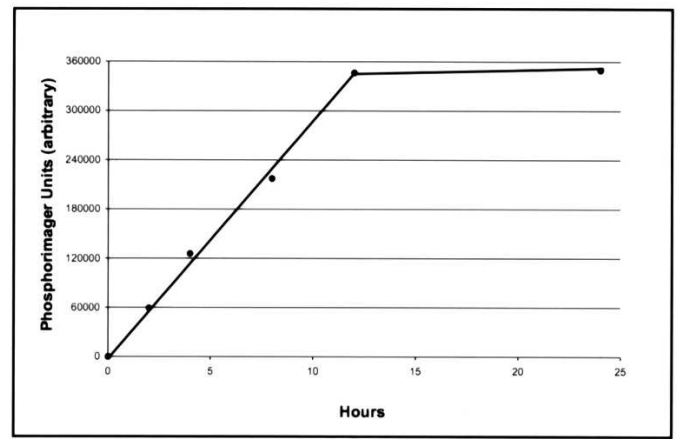

Figure 1. MtnA as a model for activated transcription. (A) A diagram of the single-copy MtnA gene located on Chromosome 3. It contains a TATA element and an initiator, although there is no DPE or MTE detectable by sequence comparison. (B) Quantitative PCR on cDNA prepared from S2 cells unchallenged $(-)$ or challenged $(+)$ with copper for $6 \mathrm{~h}$ at $25^{\circ} \mathrm{C}$. The amount of MtnA transcript is normalized to the Rp49 gene for the large ribosomal protein 32 . The ratio of MtnA/Rp49 is plotted. (C) Primer extension analysis on $20 \mu \mathrm{g}$ of total RNA from S2 cells unchallenged $(-)$ or challenged $(+)$ with copper for $6 \mathrm{~h}$ at $25^{\circ} \mathrm{C}$. Transcription initiation is mapped to a single core promoter indicated by the arrow. (D) Primer extension was performed on $12 \mu \mathrm{g}$ of total RNA before or after addition of copper, and the amount of MtnA transcript was quantitated by measuring the amount of primer extended.

adjacent metal response elements (MRE) 50 bp upstream of the TATA-box (Zhang et al. 2001). Quantitative PCR (qPCR) analysis of the endogenous gene in Drosophila S2 cells shows that the gene is highly induced $(\sim 250$-fold) after a short exposure to copper. The total amount of stable MtnA mRNA approximates the level of the abundant transcript for the ribosomal subunit Rp49 (Fig. 1B). Primer extension analysis confirms that transcriptional activation of the endogenous MtnA gene originates from 
Marr et al.

a unique start site overlapping the core promoter (Fig. 1C). The transcript accumulates linearly for $\sim 12 \mathrm{~h}$, thus measurements in this time window likely reflect relative levels of transcription of the MtnA gene (Fig. 1D). Importantly, induction at the endogenous chromosomal locus is easily assayed in order to measure physiologically relevant transcriptional activation in the context of native chromatin. Taken together, these properties establish the endogenous MtnA gene as a useful model for studying transcriptional mechanisms governing an inducible gene.
Both TFIID and Mediator are recruited to endogenous MtnA by MTF-1

Using chromatin immunoprecipitation (ChIP), we find that the sequence-specific DNA-binding protein MTF-1 is specifically recruited to the MtnA promoter region in response to copper. Curiously, when we compare the ChIP of the promoter region to a region $1 \mathrm{~kb}$ downstream, we find a significant amount of MTF-1 present on the promoter even in the absence of added copper (Fig. 2A). Under these conditions, we detect little transcrip-

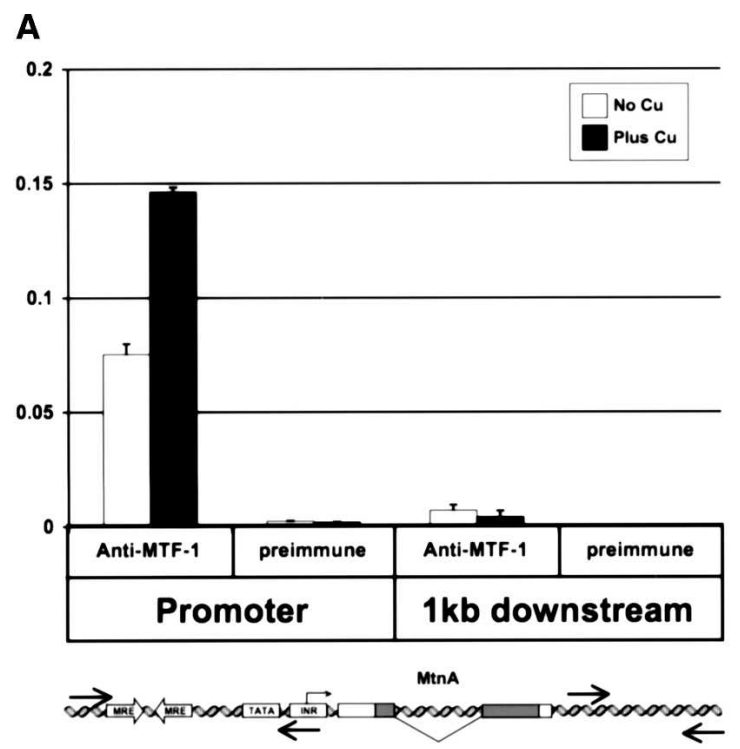

B

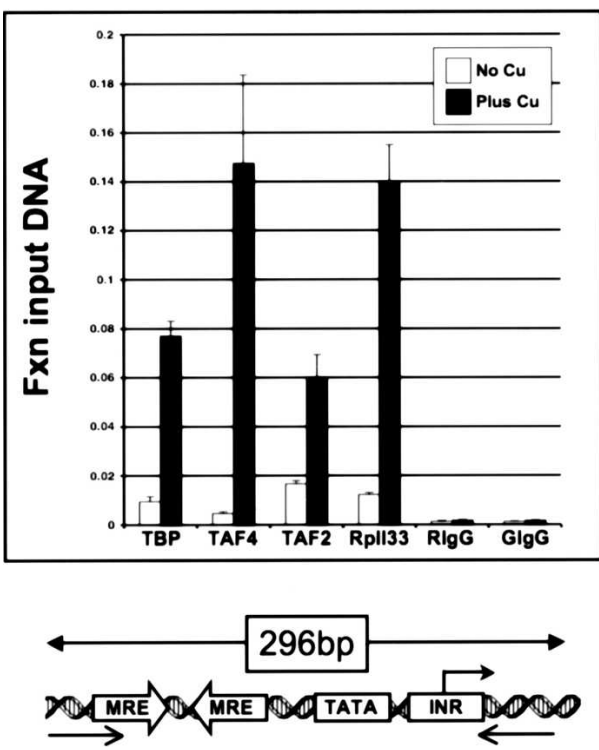

C

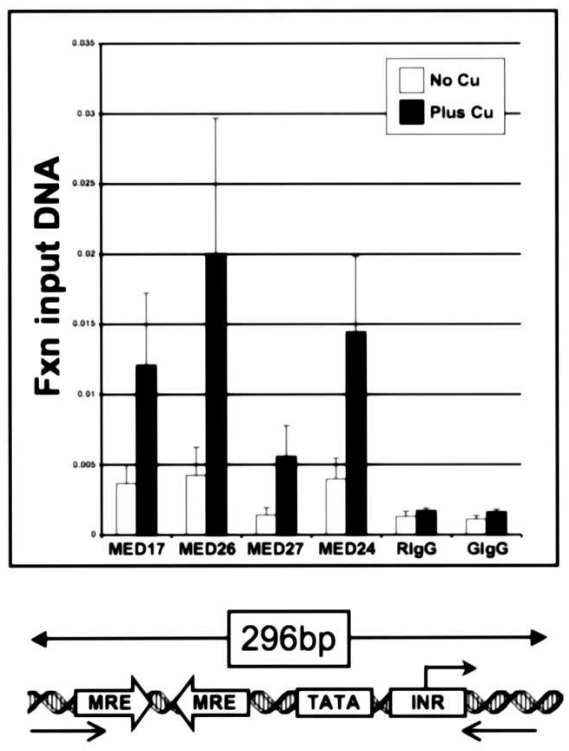

Figure 2. MTF-1, TFIID, and MED are recruited to the MtnA gene. $(A-C)$ ChIP performed as described in the Materials and Methods section. Polyclonal antibodies used for immunoprecipitation are indicated below each column. Preimmune sera, normal rabbit IgG (RIgG), and normal guinea pig IgG (GIgG) serve as negative controls. White bars represent results from untreated S2 cells, and black bars represent results from S2 cells treated with copper for $6 \mathrm{~h}$ at $25^{\circ} \mathrm{C}$. A diagram of the region amplified and the position of the primers used are shown below the graph. 
tion from this gene (Fig. 1B,C). As a preliminary experiment to investigate a potential functional interaction between TFIID and MED, we first asked whether the two complexes are both recruited in a signal-dependent manner to the MtnA gene. Using ChIP, we find that both TBP and the TAFs are efficiently recruited to the promoter region in response to copper (Fig. 2B). In addition, the MED17, MED24, MED26, and MED27 subunits of MED are all recruited to the promoter region in response to copper treatment (Fig. 2C). Consistent with the high level of induction, RNAPII occupancy at the MtnA promoter is also increased in response to heavy metal treatment (Fig. 2B). Thus, both core coactivator complexes and RNAPII are efficiently recruited to the promoter region upon induction and resultant binding of MTF-1 to the MREs.

\section{A single-cell transgenic model for studying MtnA transcriptional induction}

Because the ChIP assay is limited to measuring response in a heterogeneous population of cells, we established a transgenic model system in Drosophila S2 cells in order to visualize the response at the single-cell level. Such an approach has proved useful in understanding transcription factor dynamics in vivo (McNally et al. 2000; Tsukamoto et al. 2000; Muller et al. 2001; Janicki et al. 2004). By selecting for stably transfected MtnA firefly luciferase reporters, we generated a concatenated transgenic locus in a clonal line of S2 cells (Fig. 3A). The transgenic locus was assayed for dependence on copper using a luciferase assay (Fig. 3B). Importantly, transcription initiates a unique site that maps to the correct start
A

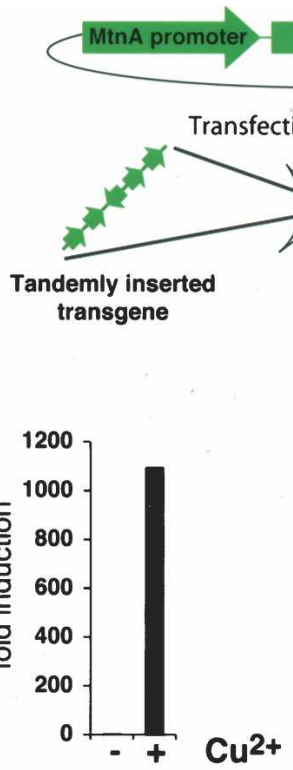

D
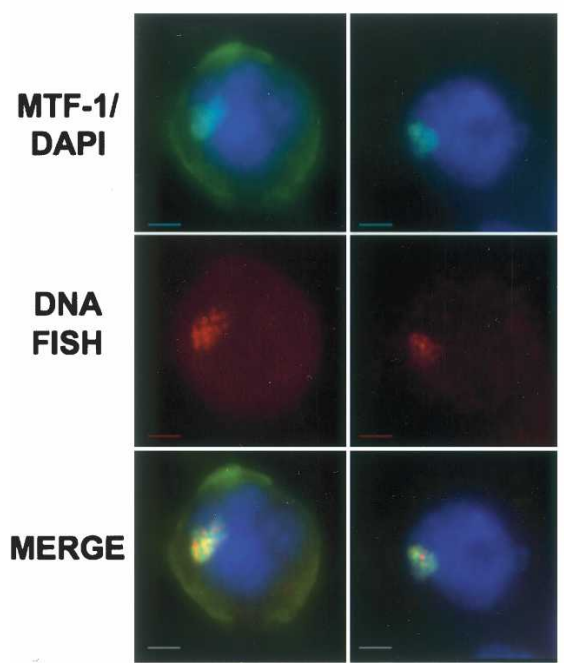

luciferase

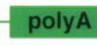

Drug resistant marker (1/20 of reporter plasmid)

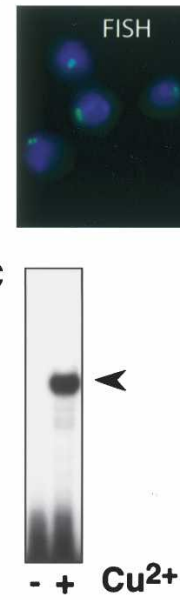

\section{Uninduced Induced}

MTF-1/ DAPI

\section{E}

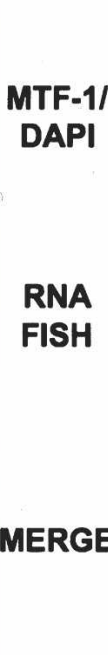

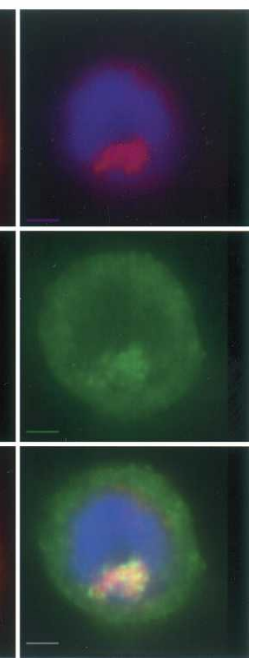

Figure 3. Single-cell model of MtnA gene activation. (A) Schematic for the generation of transgenic cell lines. The reporter plasmid is transfected into S2 cells together with a small amount of hygromycin-resistant marker plasmid. After the generation of stable cell lines, fluorescence in situ hybridization (FISH) was conducted to confirm the integration of reporter plasmids. $(B)$ Luciferase assay conducted to confirm that transcription from the transgenes exhibits low basal activity but is highly inducible upon induction by copper. (C) Primer extension assay was conducted using $20 \mu \mathrm{g}$ of total RNA from uninduced $(-)$ and induced $(+)$ transgenic cells. A single major transcription start site was observed upon induction. $(D)$ These panels show FISH experiments testing the size and response of the MtnA transgenic cluster. The top row shows images of MTF-1 (green on the left, red on the right) and DNA staining (DAPI). The middle panels mark the transgenic cluster with DNA FISH (red) on the left and RNA FISH (green) on the right. The bottom row shows the merge of the two images. MTF-1 is recruited to the transgenic cluster upon induction with copper (bottom right), and the cluster is active only upon induction (bottom left). 
site of the MtnA core promoter (Fig. 3C). With this substantial increase in gene number $(-2000)$ at the integrated transgenic locus, we should now be able to visualize direct recruitment of specific transcription factors to the MtnA promoter within a single cell.

As expected, in the absence of heavy metal, MTF-1 is predominantly cytoplasmic (Smirnova et al. 2000); however, in agreement with our ChIP data above, some MTF-1 can be detected at the transgenic cluster even in the absence of a metal stimulus (Fig. 3D). Thus, antibody labeling of MTF-1 provides a useful marker for the subnuclear location of the transgene cluster in both induced and uninduced cells. Notably, the locus is not undergoing transcription (as detected by RNA FISH) (Fig. 3D) in the absence of heavy metal induction despite the presence of some MTF-1 at the transgene cluster. Upon copper induction, MTF-1 vacates the cytoplasm and accu- mulates selectively at the transgenic locus. Under these same conditions, we also detect TBP actively recruited to this cluster (Fig. 4A). Consistent with not only TBP but holo-TFIID complex recruitment, we found that TAF2 also accumulates at the transgene (Fig. 4B). We likewise detect MED components recruited to the transgene using antibodies against MED26 (Fig. 4C). As expected, RNAPII is recruited to the cluster in a copperdependent manner consistent with the transcriptional induction of the transgene under these conditions (Fig. 4D). In contrast, TBP-related factor 1 (TRF1), a subunit known to be a key component of the RNA polymerase III core promoter recognition complex (Takada et al. 2000), is not recruited to the transgene. This negative control helps us rule out the possibility that the tandemly reiterated transgene is simply nonspecifically attracting transcription factors (Fig. 4E).
A

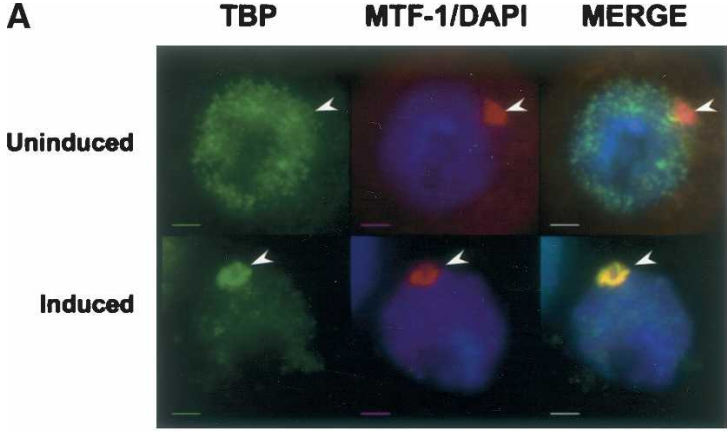

C

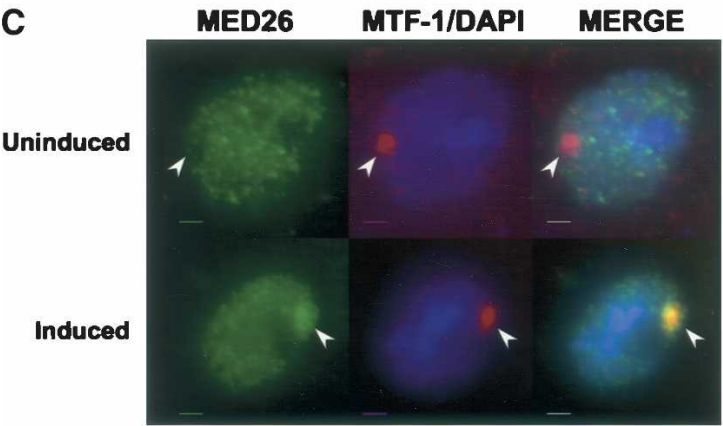

B

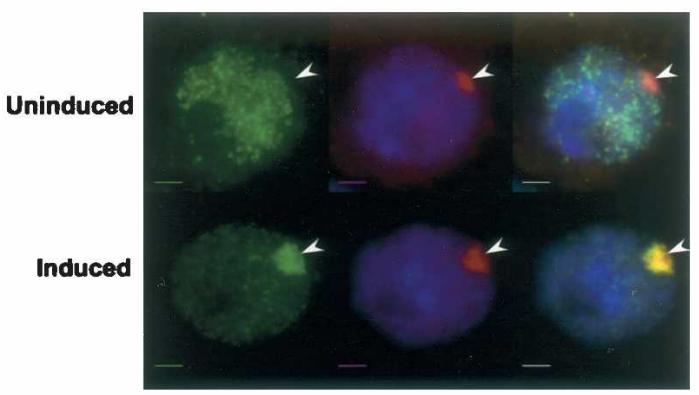

D

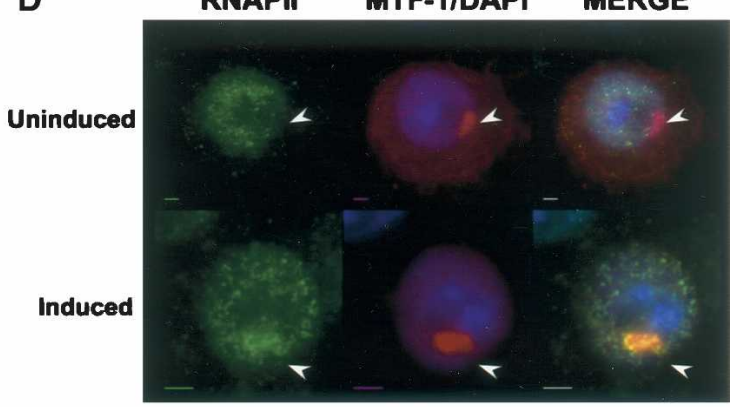

E TRF1 MTF-1/DAPI MERGE

Uninduced

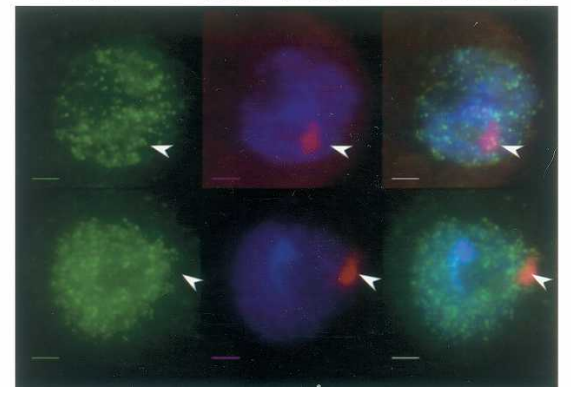

Figure 4. Recruitment of coactivators to the transgenic locus. $(A-E)$ In situ staining to determine protein recruitment to the transgenic cluster. The protein specified above the first column in each panel was detected with an FITC conjugated secondary antibody. In the middle column of each panel, MTF-1 was detected with a Rhodamin-X conjugated secondary antibody, and DNA was stained with DAPI. The third column shows the merge of these channels. In each panel, the top row shows a cell in the absence of copper; the bottom row shows a cell in the presence of copper for $2 \mathrm{~h}(A, B, D, E)$ or $4 \mathrm{~h}(C)$. Scale bar, $2 \mu \mathrm{m}$. 
RNAi depletion of coactivator complexes reveals differential roles

Having established by two independent methods that both TFIID and MED complexes are recruited to the MtnA promoter in an activator-dependent manner, we next investigated their role in potentiating transcriptional activation of the endogenous MtnA gene. We used the efficient technique of RNAi in Drosophila S2 cells to knock down expression of TFIID and MED subunits. In addition, we knocked down the activator MTF-1 to ascertain the extent of the activator's role in induction. After treatment with copper, we purified total RNA from dsRNA treated and untreated S2 cells and then assayed them by two independent methods. First, we used a primer extension analysis on equivalent amounts of total RNA. This assay revealed that we are detecting an accurate transcription from one distinct core promoter start site (Fig. 5A,E). Next, we used qPCR normalized to the Rp49 mRNA, to confirm that there is
A

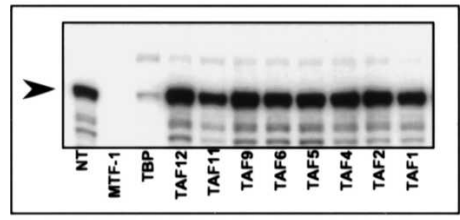

C

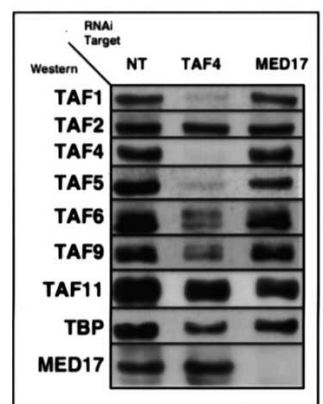

B

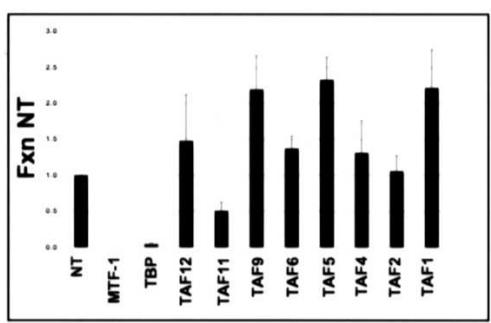

D

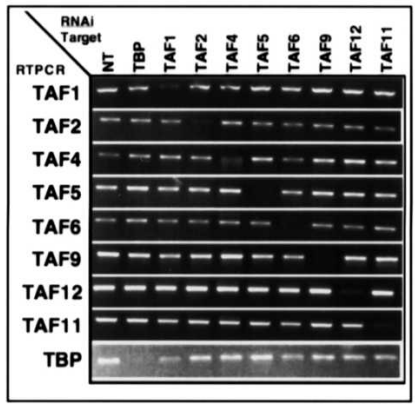

E

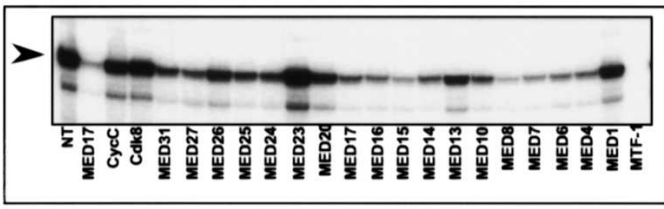

$\mathbf{F}$

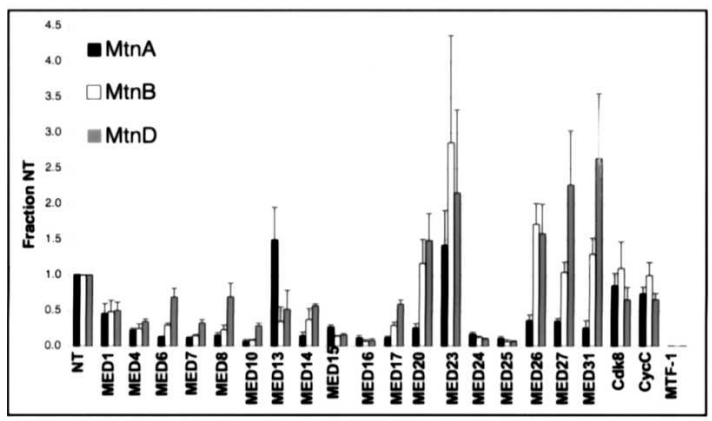

G

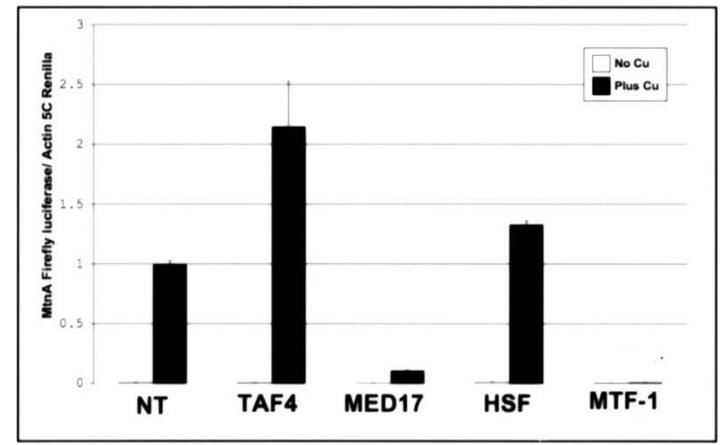

Figure 5. RNAi of coactivators reveals distinct roles for both TFIID and MED. (A) S2 cells were treated with dsRNA directed against the TFIID subunit indicated below each lane. Subsequently they were treated with copper for $6 \mathrm{~h}$. Twenty micrograms of total RNA was subjected to primer extension analysis with a primer that anneals to the endogenous MtnA transcript; the arrowhead marks the major start site of transcription. (B) The MtnA RNA level was normalized to the amount of endogenous Rp49 transcript. The data are plotted as the fraction of the level in the non-RNAi-treated cells. The TFIID subunit targeted by dsRNA is indicated below each bar. $(C)$ Protein immunoblot analysis of nuclear extract from S2 cells treated with dsRNA. The RNAi target is listed across the top of the panel. Twenty micrograms of nuclear extract was resolved by SDS-PAGE and transferred to nitrocellulose, and proteins were detected with antibodies directed against the TFIID subunits listed on the left. The MED17 RNAi-treated cells serve as a control for the specificity of the RNAi. (D) Reverse transcriptase PCR analysis of the RNA levels of the TFIID genes. The gene targeted by RNAi is listed across the top of the panel. The genes listed on the left were amplified with primers specific for their RNA. (E) S2 cells were treated with dsRNA directed against the MED subunit indicated below each lane. Subsequently they were treated with copper for 6 h. Twenty micrograms of total RNA was subjected to primer extension analysis with a primer that anneals to the endogenous MtnA transcript; the arrowhead marks the major start site of transcription. $(F)$ The MtnA, MtnB, and MtnD RNA level was normalized to the amount of endogenous Rp49 transcript. The data are plotted as the fraction of the level in the non-RNAi-treated cells. The MED subunit targeted by dsRNA is indicated below each bar. $(G)$ Transient transfection of the MtnA reporter in dsRNA-treated cells. HSF serves as a nonspecific control. The data are plotted as a ratio of MtnA promoter activity to Actin $5 \mathrm{C}$ promoter activity. 
little or no global disturbance of RNAPII transcription (Fig. 5B,F).

Not surprisingly, depletion of MTF-1 severely reduced transcriptional activation from the MtnA promoter, confirming the central role of this activator. RNAi directed against TBP also had a dramatic inhibitory effect. The MtnA promoter is $<10 \%$ as active when TBP levels are severely depleted. Surprisingly, knockdown of multiple TAFs had little apparent effect on the ability of MTF-1 to activate MtnA. Indeed, depletion of the TAFs actually stimulated (1.5- to 2-fold) production of RNA. With the exception of TAF11, a reduction of individual TAFs resulted in a remarkably uniform response. The reason for this uniformity became apparent when we examined the stability of the TFIID complex in the RNAi-treated cells. The overall stability of the holo-TFIID complex appears to be coupled to the stability of certain individual TAFs. In the most dramatic example, RNAi-targeted reduction of TAF4 leads to the concomitant loss of TAF1, TAF5, TAF6, and TAF9, as well as a detectable reduction in TBP (Fig. 5C). Interestingly, TAF2 and TAF11 are largely unaffected by depletion of TAF4. Similar results are observed for the other TAFs as well (K.J. Wright and R. Tjian, unpubl.). When we measure the transcript levels of the TAFs after RNAi treatment, it is clear that the loss of stability occurs at the protein level, since the transcript levels for nontargeted TAFs are unaffected. For example, when TAF4 is targeted, only the TAF4 transcript is depleted (Fig. 5D).

\section{MTF-1 requirement for MED subunits depends on the promoter}

In contrast to the TAFs, RNAi reduction of MED subunits gave striking but variable effects on the ability of MTF-1 to activate transcription from the MtnA promoter. Unlike TFIID, the response is far from uniform (Fig. 5E,F). For example, dsRNA directed against MED23 has little effect on induction of MtnA, while loss of MED17, the Drosophila SRB4 homolog, has a strong inhibitory effect. The lack of a uniform response in the MED RNAi led us to further investigate the potential differential response upon depletion of MED subunits at related promoters activated by MTF-1. As discussed above, Drosophila has four metallothionein genes that respond to heavy metals. Three of these-MtnA, MtnB, and MtnD-are active in S2 cells. All three of these genes are specifically activated by the same factor, MTF1. We therefore examined all three Mtn genes in a single experiment using qPCR (Fig. 5F). First, we confirmed that all three promoters, $M \operatorname{tn} A, M \operatorname{tnB}$, and $M \operatorname{tnD}$, require MTF-1 for induction. Remarkably, we find distinct differential requirements for MED subunits depending on the promoter. For example, loss of MED13, a subunit of the larger MED complex (ARC-L) thought to play a repressive role in transcription, is not essential for MtnA induction. In contrast, MED13 was found to be important for both MtnB and MtnD activation by MTF-1. In contrast, we see the opposite specificity with the MED26 subunit, a component of the smaller MED complex
(CRSP), thought to play predominantly a coactivator role in transcription. Interestingly, MED26 is required for full induction of the MtnA promoter but is dispensable for MTF-1 activation of the MtnB and MtnD promoters. Thus, these experiments reveal a remarkable example of differential dependence on cofactor composition even though all three promoters tested use the same activator. Apparently, the precise role of individual MED subunits depends on the promoter context and structure, despite the absence of any evidence of direct binding of DNA by the MED complex.

To help us rule out nonspecific effects on transcription such as a change in the concentration of free RNA polymerase, we tested representative targets from TFIID and MED in a transient transfection assay where we can normalize the effect to a second promoter. In these experiments, we chose TAF4 and MED17 as representative targets, since TAF4 compromises much of the TFIID complex and MED 17 is likely a component of the core MED complex. The transient transfection data are largely consistent with the data generated at the endogenous locus and at the transgene (Fig. 5G).

The data presented above suggest that activation of the MtnA gene requires specific MED subunits, and at the same time the TAFs appear to be playing a potential negative regulatory role. Because it is clear that the TAFs are specifically recruited in S2 cells to the MtnA promoter in a copper-dependent manner by MTF-1, we next examined whether TFIID recruitment can occur in the absence of the MED complex. To achieve this, we used RNAi directed against MED17, which results in an almost complete loss of MED activity. Surprisingly, TFIID is still efficiently recruited to the MtnA gene. ChIP experiments confirmed that TBP and TAF2 are still actively (and likely directly) recruited to the endogenous MtnA gene by MTF-1 (Fig. 6A) even when the gene is transcriptionally inactive as measured by qPCR analysis (Fig. 6B). We next used the MtnA luciferase transgene system to investigate this relationship at the single-cell level. Figure 6C shows in situ immunofluorescence analysis after the cells have been induced with copper. The first panel shows cells that have not received any dsRNA (NT), and, as expected, TBP, TAF2, and RNAPII are all recruited to the transgene. In agreement with the ChIP data above, the second panel shows that even in the absence of MED activity, after MED17 depletion, TBP and TAF2 are nevertheless efficiently recruited to the transgene. In contrast, no RNAPII can be detected at the transgene consistent with the loss of transcription activation. Apparently, TFIID is recruited to the promoter, but the promoter is not active in supporting transcription. Importantly, the third panel shows that recruitment of this "inactive TFIID" is dependent on the activator MTF-1. In the absence of MTF-1, no TFIID or RNAPII is recruited to the transgene.

This perplexing result of recruiting an apparently "inactive" TFIID prompted us to examine what happens when we deplete both TAFs and MEDs. As the Western blot in Figure 7A shows, we were able to efficiently deplete both TAF4 and MED17 to $<10 \%$ normal levels in 
A

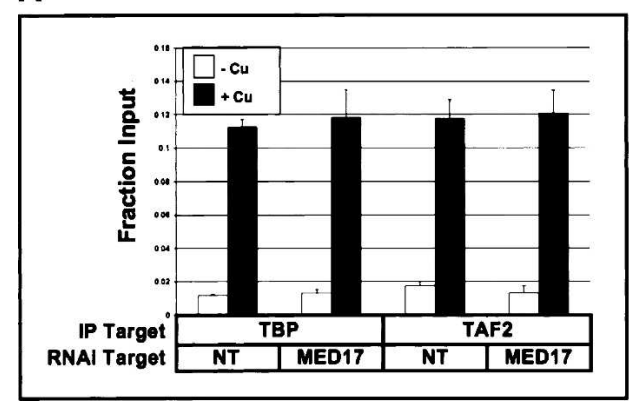

B

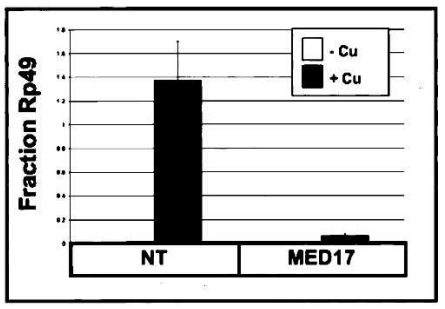

C

NT

NT
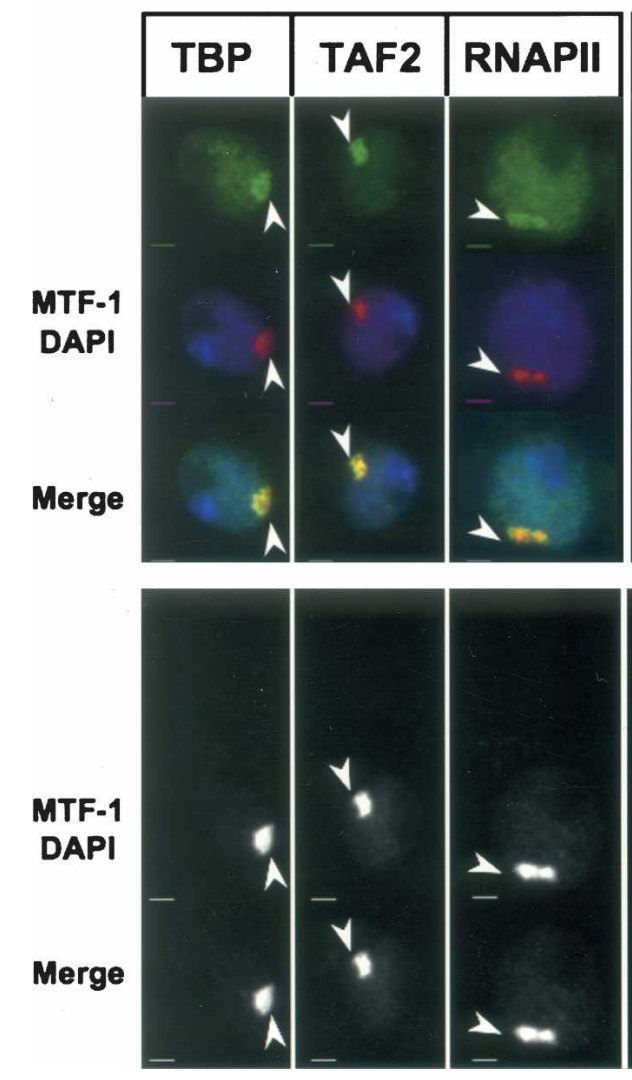

MED17 RNAi
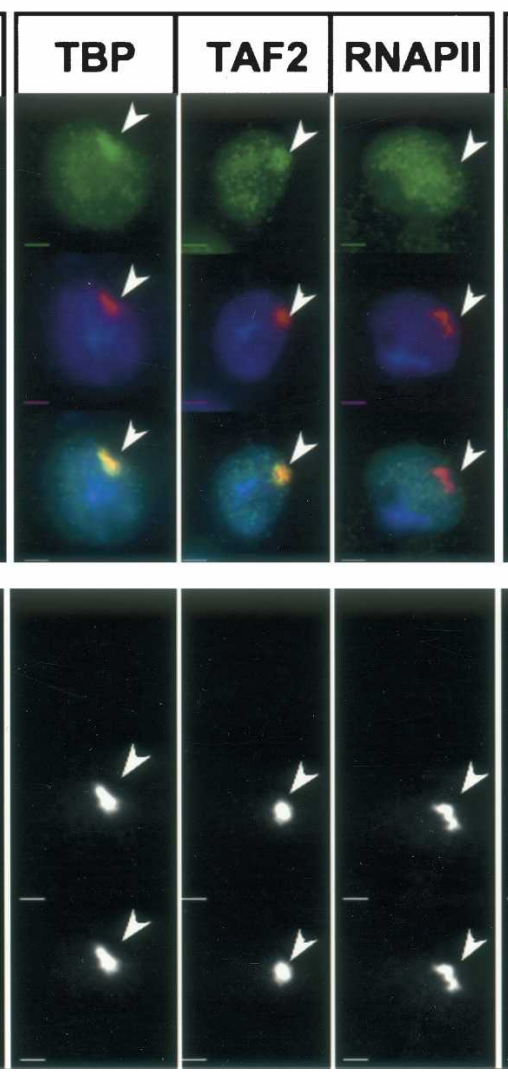

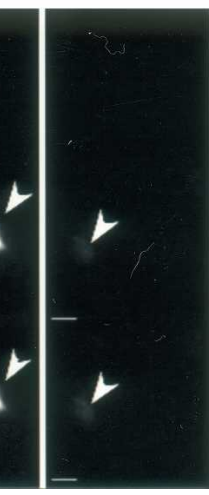

MTF-1RNAi
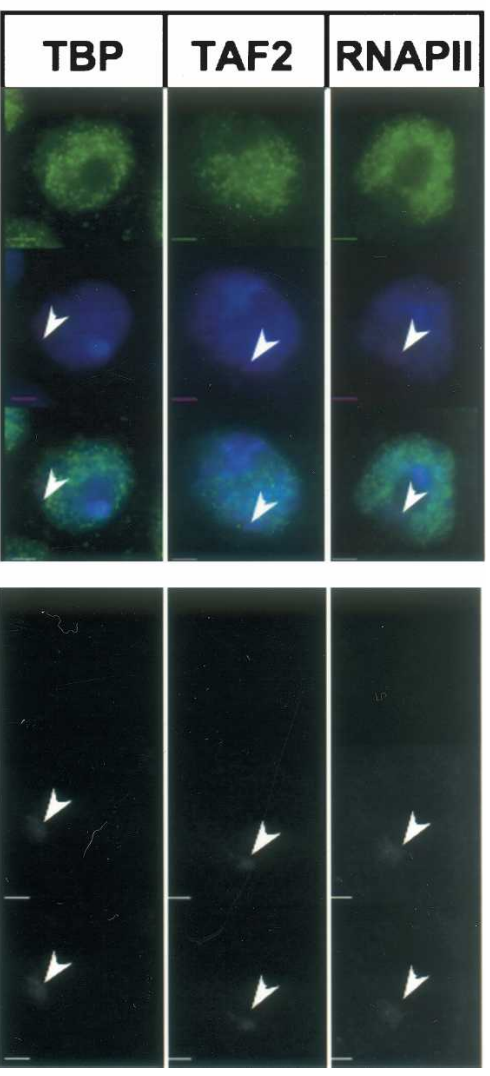

Figure 6. Independent recruitment of TFIID to MtnA transgene by MTF-1. (A) ChIP analysis of TFIID recruitment in the absence of MED. S2 cells were treated with dsRNA as indicated at the left and subsequently immunoprecipitated with the antibody indicated below the graph. White bars represent results from untreated S2 cells, and black bars represent results from S2 cells treated with copper for $6 \mathrm{~h}$ at $25^{\circ} \mathrm{C} .(B)$ Quantitative PCR analysis of the MtnA mRNA, as in Figure 1B. (C) S2 cells harboring the MtnA transgene treated with dsRNAs against MED17 and MTF-1 were induced with $\mathrm{CuSO}_{4}$ for $2 \mathrm{~h}$ and were examined for TFIID and Pol II recruitment using anti-TBP, anti-TAF2, and anti-RPB2. Arrows indicate the location of the transgene marked by MTF-1. The bottom set of black and white panels shows the MTF-1 channel only to illustrate the residual amount of MTF-1 signal after RNAi treatment used to estimate the location of the transgene. Scale bar, $2 \mu \mathrm{m}$.

the same cells. This extensive depletion is a prerequisite for interpretation of the double knockdown by RNAi. We wanted to ensure that most cells in the experiment have lost both coactivators and that we were not examining two different populations. In this double-knockdown background, we induced the cells with copper and then tested for MtnA promoter activity by both primer extension and qPCR (Fig. 7B,C). Remarkably when both the TAFs and MED complex are depleted and "removed" from the MtnA promoter, MTF-1-dependent activation of transcription is restored to $\sim 95 \%$ the level of untreated cells, which is well above the inhibited level observed when the MEDs alone are depleted (10\%-20\% residual activity) (Fig. 5C). In humans and Drosophila, TAFs can be subunits of other complexes such as TFTC and STAGA (Wieczorek et al. 1998; Kusch et al. 2003), so it is possible that the functional interaction we have analyzed is not TFIID-specific. To test this, we targeted spe- 
A

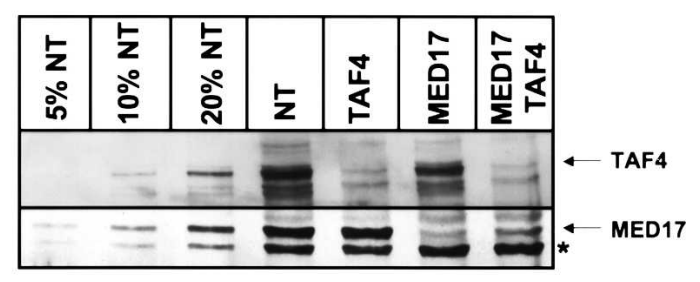

B

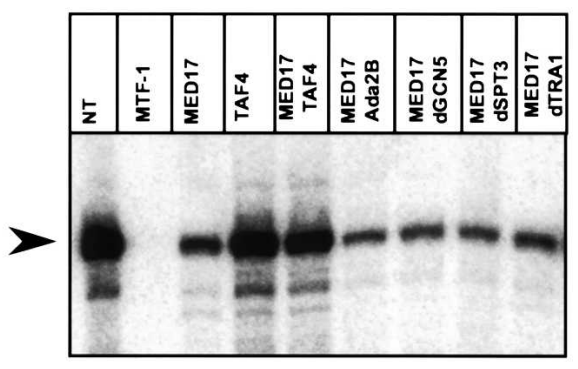

C

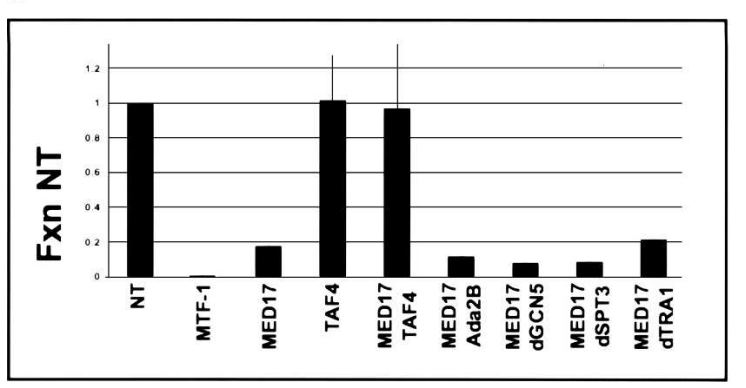

Figure 7. Depletion of TFIID suppresses depletion of MED. (A) Western analysis of nuclear extracts of S2 cells treated with dsRNA directed against either TAF4 (TFIID) or MED17, or both. Nontreated S2 cell extracts were serially diluted to provide an estimate of the depletion efficiency. Positions of TAF4 and MED17 are indicated by arrows. The asterisk denotes a crossreacting band in the anti-MED17 sera. (B) Primer extension analysis (as in Fig. 3A) of S2 cells treated with dsRNA against the subunits or combinations of subunits indicated above each lane. (C) Quantitative PCR analysis of MtnA transcripts (as in Fig. 3B) of S2 cells treated with dsRNA against the subunits or combinations of subunits indicated below each column.

cific subunits of these other complexes to determine if they would have a similar ability to rescue the MED knockdown. Unlike the TFIID subunits, RNAi against dAda2b, dGCN5, dSPT3, and dTRA1 was unable to rescue the loss of the MED subunits. These findings taken together suggest that most likely the functional relationship revealed by these experiments with the MtnA promoter, indeed, involve some regulatory transaction between TFIID and MED.

\section{Discussion}

The requirement for coactivator complexes mediating transcriptional responses to activators has been well documented (Lemon and Tjian 2000). However, by using an inducible Drosophila gene as a model system, we have uncovered a previously unknown functional interaction between two coactivator complexes, TFIID and MED. In the absence of TAFs, the cell responds inappropriately to a metal stimulus. The cell synthesizes $50 \%-$ $200 \%$ more mRNA from the MtnA gene than it does in the presence of the TAFs. Our data suggest that at this gene, TFIID is recruited in an inactive state, a state that impedes initiation of transcription. We believe this sets up a checkpoint early in the initiation process to meter the RNA synthesis. The MED complex must be recruited to get past this checkpoint. We postulate that the MED complex likely modifies TFIID, converting it to an active state. This could be accomplished either through one of the known enzymatic activities of MED, phosphorylating (cdk8) (Sun et al. 1998; Liu et al. 2004) or ubiquitylating (MED8) (Brower et al. 2002) TFIID subunits, or through some, as yet undetected, chaperonelike function that remodels TFIID into an active conformation. Not surprisingly then, in the absence of MED subunits the cell cannot mount an appropriate response to environmental signals. In fact, depletions of many of the MED subunits lead to $<20 \%$ of the normal amount of mRNA. Unlike the uniform response to depletion of TAFs, the response to depletion of MEDs is much less uniform. One possibility is that the MED complex is more functionally and structurally diverse than TFIID. Indeed, alternative subcomplexes of MED have been purified biochemically (Boyer et al. 1999; Ryu et al. 1999; Malik et al. 2000; Taatjes and Tjian 2004), whereas no such subcomplexes of TFIID have been reported.

By analysis of three different Mtn genes, all of which are dependent on the same single activator, we find surprisingly that there is a differential requirement of specific MED subunits at the three Mtn promoters. We take this as evidence that, depending on the precise arrangement of cis elements and promoter context, the same activator can require different mediator subunits or modules to transmit its signals to the basal apparatus.

Interestingly, the kinase module of the MED complex, previously linked with repression functions, is required for efficient activation at two of the promoters. This result, combined with the finding that at the MtnA promoter the TAFs have a repressive regulatory influence on transcription initiation, underscores the difficulty in assigning black and white functions to the coactivator complexes. It is likely that both TFIID and MED interpret multiple inputs from cellular signals and act either positively or negatively depending on the signals received as well as the specific promoter context. As such, the complexes may better be viewed as coregulators since they can play either a positive or negative role in the process of modulating gene expression. For example, only when both TFIID and MED are intact do Drosophila S2 cells produce the appropriate amounts of MtnA mRNA. In contrast, when either coactivator complex is disrupted, we see aberrant levels of transcription. However, when both coactivator complexes are depleted, a significant level of metal inducible activation is actually restored. Presumably, in this "stripped down" system, 
some portion of the remaining TBP pool can mediate transcription. Curiously, in the absence of TAFs but with a full complement of MEDs, there is also an aberrant level of transcription consistent with the notion that there is some finely tuned codependence between the TBP/TAF complex and the MED complex at this promoter.

Our results also reinforce the notion that the activator is the primary determinant of the transcriptional response. The MTF-1 depletion experiments were the most detrimental to mRNA induction. In the absence of MTF-1, there is no detectable activation of the Mtn genes. In contrast, there is some residual transcription of MtnA even when either the MEDs or TBP are largely depleted from the Drosophila cells. This remaining activity could be due to incomplete depletion, or it could indicate alternative mechanisms of activation that are activator-dependent but can partially bypass the requirement for the coregulator complexes.

In the course of testing the requirement for TAFs in activated transcription, we discovered the codependent stability of the TFIID complex. Particularly striking is the finding that TAF4 depletion destabilizes most of the other TAFs and, to some extent, even TBP. Therefore, the TAF depletion experiments most likely reflect a loss of holo-TFIID rather than just the loss of individual subunits. It is worth noting that metazoan organisms contain multiple variants of TAF4: TAF4b in vertebrates and no-hitter in Drosophila (Dikstein et al. 1996; Freiman et al. 2001; Hiller et al. 2004). Both of these have been implicated in tissue-specific gene expression. It is conceivable that substitution of this keystone TAF can provide a mechanism to change the entire coregulator profile of TFIID.

One intriguing question this work raises is: Why would an activator recruit an inactive TFIID complex to the promoter? There are several previously described cases in which TFIID occupancy at a promoter does not strictly correlate with transcriptional activity (Breiling et al. 2001; Chen et al. 2002; Dellino et al. 2004; Wang et al. 2005). However, in most of these cases the genes being examined were either in a repressed or an unstimulated state. In contrast, our studies were designed to specifically measure the role of coactivator complexes such as TFIID and MED in the context of an active gene MtnA upon metal stimulation. Our ability to deplete MED activity under these conditions revealed the unexpected finding that although TFIID is dynamically recruited to the MtnA promoter, TFIID is mainly held in an "inactive" state until the second cofactor complex, MED, is recruited. Perhaps this recruitment of an "inactive" TFIID is a more common phenomenon that can only be detected in special circumstances and may represent a previously unappreciated control mechanism in transcription activation. If the activator first recruits TFIID, then subsequently recruits MED, and there is a requirement for additional factors to potentiate the secondary recruitment of coregulator assemblies, then this provides a potential checkpoint for fine-tuning the control of gene expression. Alternatively, since the cell invests a significant amount of energy in making a high level of transcript, requirement of continued stimulation (i.e., activator bound at the promoter) for mRNA production would provide the most economical use of resources.

\section{Materials and methods}

\section{Cell culture and RNAi}

Drosophila Schneider line 2 cells (S2) cells were maintained at $25^{\circ} \mathrm{C}$ in M3BPYE (DGRC). RNAi was carried out essentially as described (Clemens et al. 2000) using 15-40 $\mu \mathrm{g}$ of dsRNA. For copper induction, cells were treated with $0.5 \mathrm{mM} \mathrm{CuSO}_{4}$ for $6 \mathrm{~h}$ at $25^{\circ} \mathrm{C}$. For the transient transfection experiments, cells were treated with dsRNA as described except that addition of the complete medium contained transfection reagents. Three days after transfection, copper was added for $6 \mathrm{~h}$ and luciferase assays were performed using the dual luciferase assay system (Promega) according to the manufacturer's directions. Transfection was performed using Effectene (QIAGEN) according to the manufacturer's instructions, as modified for S2 cells.

\section{ChIP}

ChIP was carried out essentially as described (Puig et al. 2003) for MTF-1. MED, TFIID, and Pol II experiments were carried out as above except that the cells were first treated with $15 \mathrm{mM}$ DMP as described (Kurdistani and Grunstein 2003). The amount of DNA precipitated was determined by quantitative PCR using an Opticon 4 and the QIAGEN SYBER green kit.

\section{Primer extension and quantitative PCR}

Total RNA was purified using TRI-reagent (Sigma) following the manufacturer's recommendations. For quantitative PCR, the RNA was further treated with DNase I (Ambion) for $1 \mathrm{~h}$ at $37^{\circ} \mathrm{C}$ and further purified with RNeasy columns (Qiagen). cDNA was synthesized from DNase I-treated RNA with Superscript II and random hexamers following the manufacturer's recommendations (Invitrogen). The RNA level was determined using an Opticon 4 quantitative PCR instrument and the QIAGEN SYBER green kit with Rp49 as the control. Primer sequences are available upon request.

\section{Generation of S2 cells harboring transgenic MtnA reporter cassette}

The region -367 to +56 relative to the start site of transcription of MtnA promoter was amplified by PCR and inserted in the pGL3 basic vector (Promega). This construct was used to transfect S2 cells together with $1 / 20$ of pCoHygro vector (Invitrogen) by calcium phosphate method. The hygromycin-resistant clones were isolated and tested for inducibility by copper and nuclear localization by FISH. FITC and Alexa568 conjugated probes for DNA FISH were generated first by fragmenting pGL3 basic plasmids with AciI, AluI, HaeIII, MseI, RsaI, and Sau3AI and directly conjugating fluorophores using ULYSIS Nucleic Acid Labeling Kits (Molecular Probes). For RNA FISH, probes were synthesized using the DIG-Nick Translation Mix (Roche). The luciferase assays shown in Figure 3B were conducted using cell extracts derived from $16 \mathrm{~h}$ of induction with $500 \mu \mathrm{M}$ $\mathrm{CuSO}_{4}$. For the primer extension analysis of transgenic mtnA genes, we used a primer annealing to luciferase transcripts (AC CAACAGTACCGGAATGCCAAG) and $20 \mu \mathrm{g}$ of total RNA isolated from transgenic cells treated with $500 \mu \mathrm{M} \mathrm{CuSO}_{4}$ 
for $24 \mathrm{~h}$. Subsequently, the isolated clone was transfected with a Flag-tagged MTF-1 expression vector using Effectene (Qiagen), and stable cells were selected by addition of blasticidin (Invitrogen) to the media. The number of copies in the transgene was estimated by quantitative PCR of the MtnA promoter compared to the single-copy Actin 5C promoter. Serial dilutions of genomic DNA from the transgenic clone and the parental line were analyzed for the number of MtnA promoters normalized to the number of Actin 5C promoters. The ratio of MtnA to Actin $5 \mathrm{C}$ in the parental line was taken as 1.

\section{Microscopy}

S2 cells grown on a coverslip were fixed with $4 \%$ formaldehyde in $1 \times$ PBS for $10 \mathrm{~min}$ and permeabilized with $1 \times$ PBS containing $0.1 \%$ Triton X-100 for 15 min. After incubation with primary antibody for $3 \mathrm{~h}$ and secondary antibodies (Rhodamin X conjugated anti-mouse, FITC conjugated anti-goat, and FITC conjugated anti-rabbit antibodies; Jackson ImmunoResearch Laboratories) for $45 \mathrm{~min}$, the cells were mounted on slides. DNA and RNA FISH combined with antibody detection of MTF-1 was carried out essentially using the method described (Tam et al. 2002). All the images were captured using a Deltavision microscope (Applied Precision).

\section{Antibodies}

Anti-TBP antibody was raised against full-length Drosophila TBP. The antibody against TAF2 was previously described (Verrijzer et al. 1994), and anti-Pol II (RPB2) antibody and anti-TRF1 antibody were provided by A. Greenleaf (Duke University, Durham, NC) and S. Takada (MD Anderson Cancer Research Center, University of Texas, Houston, TX), respectively. AntiRpII33 antibody was kindly provided by J. Lis (Cornell University, Ithaca, NY). All the antisera used for microscopy were antigen-affinity purified and used for cell staining at the dilution of 1:400. Monoclonal anti-Flag M2 antibody was from Sigma. Antibodies to the TAFs were obtained from a library of monoclonal antibodies to Drosophila TFIID as previously described (Weinzierl et al. 1993). Anti-MED17 was raised against full-length Drosophila MED17 in guinea pigs. Anti-MED26 was raised against the first 100 amino acids of Drosophila MED26 in rabbits. Anti-MED27 was raised against full-length Drosophila MED27 in guinea pigs. Anti-MED24 was raised against a fragment of Drosophila MED24 in rabbit. Rabbit and guinea pig normal antisera were purchased from Jackson ImmunoResearch Laboratories.

\section{Acknowledgments}

We thank A. Greenleaf, S. Takada, and J. Lis for their generous gifts of anti-Pol II, TRF1, and RpII33 antibodies. We thank D. Egli and W. Shaffner for providing full-length cDNA for Drosophila MTF-1, and Z. Cande for the use of a microscope. M.T.M. was supported by a fellowship from the Damon Runyon Cancer Research Foundation (DRG-\#1684).

\section{References}

Boube, M., Joulia, L., Cribbs, D.L., and Bourbon, H.M. 2002. Evidence for a mediator of RNA polymerase II transcriptional regulation conserved from yeast to man. Cell 110: $143-151$.

Boyer, T.G., Martin, M.E., Lees, E., Ricciardi, R.P., and Berk, A.J. 1999. Mammalian Srb/Mediator complex is targeted by ad- enovirus E1A protein. Nature 399: 276-279.

Breiling, A., Turner, B.M., Bianchi, M.E., and Orlando, V. 2001. General transcription factors bind promoters repressed by Polycomb group proteins. Nature 412: 651-655.

Brower, C.S., Sato, S., Tomomori-Sato, C., Kamura, T., Pause, A., Stearman, R., Klausner, R.D., Malik, S., Lane, W.S., Sorokina, I., et al. 2002. Mammalian mediator subunit mMED8 is an Elongin BC-interacting protein that can assemble with $\mathrm{Cul} 2$ and Rbx1 to reconstitute a ubiquitin ligase. Proc. Nat1. Acad. Sci. 99: 10353-10358.

Chen, D., Hinkley, C.S., Henry, R.W., and Huang, S. 2002. TBP dynamics in living human cells: Constitutive association of TBP with mitotic chromosomes. Mol. Biol. Cell 13: 276-284.

Clemens, J.C., Worby, C.A., Simonson-Leff, N., Muda, M., Maehama, T., Hemmings, B.A., and Dixon, J.E. 2000. Use of double-stranded RNA interference in Drosophila cell lines to dissect signal transduction pathways. Proc. Natl. Acad. Sci. 97: 6499-6503.

Dellino, G.I., Schwartz, Y.B., Farkas, G., McCabe, D., Elgin, S.C., and Pirrotta, V. 2004. Polycomb silencing blocks transcription initiation. Mol. Cell 13: 887-893.

Dikstein, R., Zhou, S., and Tjian, R. 1996. Human TAFII 105 is a cell type-specific TFIID subunit related to hTAFII130. Cell 87: 137-146.

Dynlacht, B.D., Hoey, T., and Tjian, R. 1991. Isolation of coactivators associated with the TATA-binding protein that mediate transcriptional activation. Cell 66: 563-576.

Egli, D., Selvaraj, A., Yepiskoposyan, H., Zhang, B., Hafen, E., Georgiev, O., and Schaffner, W. 2003. Knockout of 'metalresponsive transcription factor' MTF-1 in Drosophila by homologous recombination reveals its central role in heavy metal homeostasis. EMBO J. 22: 100-108.

Fondell, J.D., Guermah, M., Malik, S., and Roeder, R.G. 1999. Thyroid hormone receptor-associated proteins and general positive cofactors mediate thyroid hormone receptor function in the absence of the TATA box-binding protein-associated factors of TFIID. Proc. Natl. Acad. Sci. 96: 1959-1964.

Freiman, R.N., Albright, S.R., Zheng, S., Sha, W.C., Hammer, R.E., and Tjian, R. 2001. Requirement of tissue-selective TBP-associated factor TAFII105 in ovarian development. Science 293: 2084-2087.

Hiller, M., Chen, X., Pringle, M.J., Suchorolski, M., Sancak, Y., Viswanathan, S., Bolival, B., Lin, T.Y., Marino, S., and Fuller, M.T. 2004. Testis-specific TAF homologs collaborate to control a tissue-specific transcription program. Development 131: 5297-5308.

Hoffman, A., Sinn, E., Yamamoto, T., Wang, J., Roy, A., Horikoshi, M., and Roeder, R.G. 1990. Highly conserved core domain and unique $\mathrm{N}$ terminus with presumptive regulatory motifs in a human TATA factor (TFID). Nature 346: 387390.

Janicki, S.M., Tsukamoto, T., Salghetti, S.E., Tansey, W.P., Sachidanandam, R., Prasanth, K.V., Ried, T., Shav-Tal, Y., Bertrand, E., Singer, R.H., et al. 2004. From silencing to gene expression: Real-time analysis in single cells. Cell 116: 683698.

Johnson, K.M. and Carey, M. 2003. Assembly of a mediator/ TFIID/TFIIA complex bypasses the need for an activator. Curr. Biol. 13: 772-777.

Johnson, K.M., Wang, J., Smallwood, A., Arayata, C., and Carey, M. 2002. TFIID and human mediator coactivator complexes assemble cooperatively on promoter DNA. Genes \& Dev. 16: $1852-1863$.

Kim, Y.J., Bjorklund, S., Li, Y., Sayre, M.H., and Kornberg, R.D. 1994. A multiprotein mediator of transcriptional activation and its interaction with the $\mathrm{C}$-terminal repeat domain of 
RNA polymerase II. Cell 77: 599-608.

Kurdistani, S.K. and Grunstein, M. 2003. In vivo protein-protein and protein-DNA crosslinking for genomewide binding microarray. Methods 31: 90-95.

Kusch, T., Guelman, S., Abmayr, S.M., and Workman, J.L. 2003. Two Drosophila Ada2 homologues function in different multiprotein complexes. Mol. Cell. Biol. 23: 3305-3319.

Lemon, B. and Tiian, R. 2000. Orchestrated response: A symphony of transcription factors for gene control. Genes \& Dev. 14: 2551-2569.

Liu, Y., Kung, C., Fishburn, J., Ansari, A.Z., Shokat, K.M., and Hahn, S. 2004. Two cyclin-dependent kinases promote RNA polymerase II transcription and formation of the scaffold complex. Mol. Cell. Biol. 24: 1721-1735.

Malik, S., Gu, W., Wu, W., Qin, J., and Roeder, R.G. 2000. The USA-derived transcriptional coactivator PC2 is a submodule of TRAP/SMCC and acts synergistically with other PCs. Mol. Cell 5: 753-760.

Maroni, G., Otto, E., and Lastowski-Perry, D. 1986. Molecular and cytogenetic characterization of a metallothionein gene of Drosophila. Genetics 112: 493-504.

Maroni, G., Otto, E., Lastowski-Perry, D., and Price, D.H. 1987. The metallothionein gene of Drosophila. Experientia Suppl. 52: 385-392.

McNally, J.G., Muller, W.G., Walker, D., Wolford, R., and Hager, G.L. 2000. The glucocorticoid receptor: Rapid exchange with regulatory sites in living cells. Science 287: 1262-1265.

Meisterernst, M., Roy, A.L., Lieu, H.M., and Roeder, R.G. 1991. Activation of class II gene transcription by regulatory factors is potentiated by a novel activity. Cell 66: 981-993.

Muller, W.G., Walker, D., Hager, G.L., and McNally, J.G. 2001. Large-scale chromatin decondensation and recondensation regulated by transcription from a natural promoter. J. Cell Biol. 154: 33-48.

Naar, A.M., Beaurang, P.A., Robinson, K.M., Oliner, J.D., Avizonis, D., Scheek, S., Zwicker, J., Kadonaga, J.T., and Tjian, R. 1998. Chromatin, TAFs, and a novel multiprotein coactivator are required for synergistic activation by $\mathrm{Sp} 1$ and SREBP-1a in vitro. Genes \& Dev. 12: 3020-3031.

Naar, A.M., Lemon, B.D., and Tjian, R. 2001. Transcriptional coactivator complexes. Annu. Rev. Biochem. 70: 475-501.

Otto, E., Allen, J.M., Young, J.E., Palmiter, R.D., and Maroni, G. 1987. A DNA segment controlling metal-regulated expression of the Drosophila melanogaster metallothionein gene Mtn. Mol. Cell. Biol. 7: 1710-1715.

Peterson, M.G., Tanese, N., Pugh, B.F., and Tjian, R. 1990. Functional domains and upstream activation properties of cloned human TATA binding protein. Science 248: 1625-1630.

Puig, O., Marr, M.T., Ruhf, M.L., and Tjian, R. 2003. Control of cell number by Drosophila FOXO: Downstream and feedback regulation of the insulin receptor pathway. Genes \& Dev. 17: 2006-2020.

Rachez, C., Lemon, B.D., Suldan, Z., Bromleigh, V., Gamble, M., Naar, A.M., Erdjument-Bromage, H., Tempst, P., and Freedman, L.P. 1999. Ligand-dependent transcription activation by nuclear receptors requires the DRIP complex. Nature 398: 824-828.

Ryu, S., Zhou, S., Ladurner, A.G., and Tjian, R. 1999. The transcriptional cofactor complex CRSP is required for activity of the enhancer-binding protein Sp1. Nature 397: 446-450.

Sato, S., Tomomori-Sato, C., Parmely, T.J., Florens, L., Zybailov, B., Swanson, S.K., Banks, C.A., Jin, J., Cai, Y., Washburn, M.P., et al. 2004. A set of consensus mammalian mediator subunits identified by multidimensional protein identification technology. Mol. Cell 14: 685-691.
Smirnova, I.V., Bittel, D.C., Ravindra, R., Jiang, H., and Andrews, G.K. 2000. Zinc and cadmium can promote rapid nuclear translocation of metal response element-binding transcription factor-1. J. Biol. Chem. 275: 9377-9384.

Sun, X., Zhang, Y., Cho, H., Rickert, P., Lees, E., Lane, W., and Reinberg, D. 1998. NAT, a human complex containing Srb polypeptides that functions as a negative regulator of activated transcription. Mol. Cell 2: 213-222.

Taatjes, D.J. and Tjian, R. 2004. Structure and function of CRSP/Med2; a promoter-selective transcriptional coactivator complex. Mol. Cell 14: 675-683.

Taatjes, D.J., Marr, M.T., and Tjian, R. 2004. Regulatory diversity among metazoan co-activator complexes. Nat. Rev. Mol. Cell Biol. 5: 403-410.

Takada, S., Lis, J.T., Zhou, S., and Tjian, R. 2000. A TRF1:BRF complex directs Drosophila RNA polymerase III transcription. Cell 101: 459-469.

Tam, R., Shopland, L.S., Johnson, C.V., McNeil, J.A., and Lawrence, J.B. 2002. Applications of RNA FISH for visualizing gene expression and nuclear architecture. Oxford University Press, NY.

Tsukamoto, T., Hashiguchi, N., Janicki, S.M., Tumbar, T., Belmont, A.S., and Spector, D.L. 2000. Visualization of gene activity in living cells. Nat. Cell Biol. 2: 871-878.

Verrijzer, C.P., Yokomori, K., Chen, J.L., and Tjian, R. 1994. Drosophila TAFII150: Similarity to yeast gene TSM-1 and specific binding to core promoter DNA. Science 264: 933941.

Wang, G., Balamotis, M.A., Stevens, J.L., Yamaguchi, Y., Handa, H., and Berk, A.J. 2005. Mediator requirement for both recruitment and postrecruitment steps in transcription initiation. Mol. Cell 17: 683-694.

Weinzierl, R.O., Dynlacht, B.D., and Tjian, R. 1993. Largest subunit of Drosophila transcription factor IID directs assembly of a complex containing TBP and a coactivator. Nature 362: 511-517.

Wieczorek, E., Brand, M., Jacq, X., and Tora, L. 1998. Function of TAF(II)-containing complex without TBP in transcription by RNA polymerase II. Nature 393: 187-191.

Zhang, B., Egli, D., Georgiev, O., and Schaffner, W. 2001. The Drosophila homolog of mammalian zinc finger factor MTF-1 activates transcription in response to heavy metals. Mol. Cell. Biol. 21: 4505-4514. 


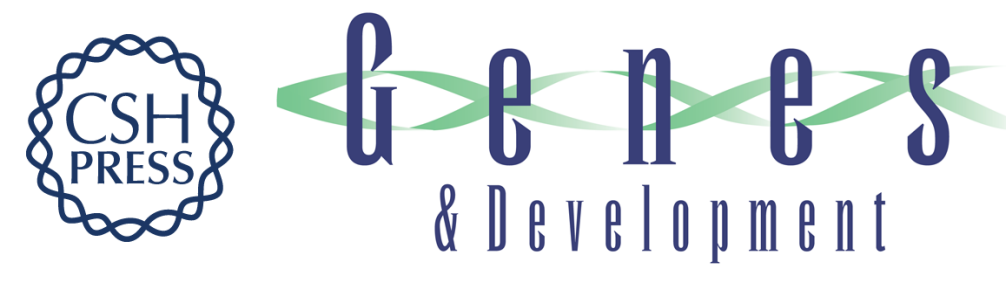

\section{Coactivator cross-talk specifies transcriptional output}

Michael T. Marr II, Yoh Isogai, Kevin J. Wright, et al.

Genes Dev. 2006, 20:

Access the most recent version at doi:10.1101/gad.1418806

References This article cites 47 articles, 21 of which can be accessed free at: http://genesdev.cshlp.org/content/20/11/1458.full.html\#ref-list-1

License

Email Alerting Receive free email alerts when new articles cite this article - sign up in the box at the top Service right corner of the article or click here.

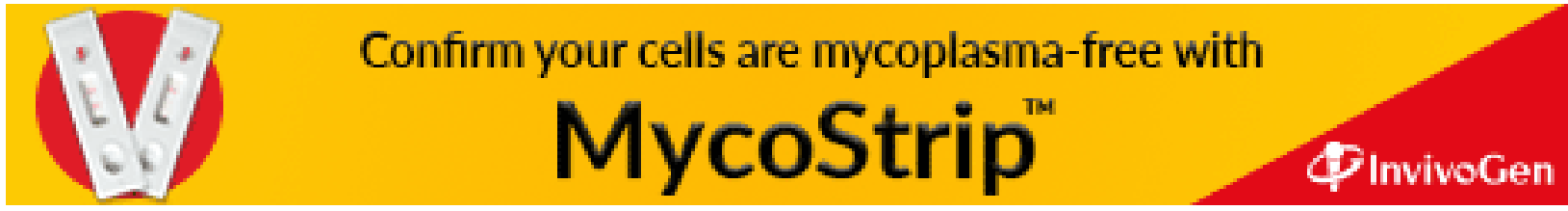

